\title{
Margin Status Cannot be Assessed
}

National Cancer Institute

\section{Source}

National Cancer Institute. Margin Status Cannot be Assessed. NCI Thesaurus. Code C160705.

A result that the marg in status cannot be assessed. 